\title{
Acknowledgment
}

Editor Thomas L. Sexton gratefully acknowledges the following individuals who served as ad hoc reviewers of manuscripts submitted to Couple and Family Psychology: Research and Practice from October 2015 to September 2016.

Nicholas Anthony

Armenti

\section{Kendra Campbell}

Adam Robert Fisher

Nathan Hardy
George Holden

Erika Lawrence

Heather MacPherson

Arthur Nielsen

Heather M. Pederson
Kelley Quirk

Jessica Rohlfing Pryor

Sandra Stith

Nona Leigh Wilson 